\title{
FOKKER-PLANCK MODELS FOR ROTATING STELLAR SYSTEMS
}

\author{
CHRISTIAN EINSEL AND RAINER SPURZEM \\ Institut für Astronomie und Astrophysik der Universität, \\ Olshausenstraße 40, D-24098 Kiel \\ e-mail: supas015@astrophysik.uni-kiel.d400.de
}

\section{Introduction}

Observations of Globular Cluster ellipticity distributions related to some fundamental parameters give strong evidence for a decay of rotational energy in these systems with time. In order to study the effectiveness of angular momentum transport (or loss, resp.) a code has been written which solves the Fokker-Planck equation in $\left(E, J_{z}\right)$-space and follows the evolution from some initial conditions through core collapse (and possibly gravothermal oscillations) up to the post-collapse phase. For the purpose of comparability with $\mathrm{N}$-body simulations rotating initial model configurations according to the prescriptions of Lupton \& Gunn (1987) have been constructed. These models are intended to continue previous work by Goodman (1983, Fokker-Planck) and Akiyama \& Sugimoto (1989, N-Body). In this contribution the derivation of the flux coefficients is given.

\section{The Flux Coefficients}

The Fokker-Planck step involves the calculation of the diffusion coefficients. Several paths for their derivation may be chosen. Here we follow the approach of Rosenbluth, MacDonald \& Judd (1957) involving covariant derivatives of tensorial objects. While we have cylindrical coordinates in real space $(\rho, z, \phi)$, spherical symmetry is applied to velocity space: $q^{1}=v ; q^{2}=v_{\psi} ; q^{3}=v_{\phi}$. After constructing the corresponding metric tensor $\left(a^{\mu \nu}\right)$, the Fokker-Planck equation is written in tensorial form:

$$
\frac{1}{\Gamma_{a}} \frac{\partial f_{a}}{\partial t}=-\left(f T_{a}^{\mu}\right), \mu_{\mu}+\frac{1}{2}\left(f S_{a}^{\mu \nu}\right)_{\mu \nu}
$$

where the commas denote covariant derivatives, the subscript $a$ particle species and the factor $\Gamma_{a}=4 \Pi G m_{a}^{2} \ln \Lambda$ contains the usual Coulomb logarythm. The diffusion coefficients, which are expressed as (covariant) derivatives of the Rosenbluth potentials, are now written as tensorial objects. We arrive at the following 
expressions for the tensors given above (note, that axial symmetry exists):

$$
\begin{gathered}
T_{a}^{1}=\frac{\partial h}{\partial v}+\frac{v_{\phi}}{v} \frac{\partial h}{\partial v_{\phi}} \quad, \quad T_{a}^{3}=\frac{v_{\phi}}{v} \frac{\partial h}{\partial v}+\frac{\partial h}{\partial v_{\phi}} \\
T_{a}^{2}=0=S^{12}=S^{21}=S^{23}=S^{32} \\
S^{11}=\frac{\partial^{2} g}{\partial v^{2}}+2 \frac{v_{\phi}}{v} \frac{\partial^{2} g}{\partial v \partial v_{\phi}}+\frac{v_{\phi}^{2}}{v^{2}} \frac{\partial^{2} g}{\partial v_{\phi}^{2}} \quad, \quad S^{22}=\frac{1}{v\left(v^{2}-v_{\phi}^{2}\right)} \frac{\partial g}{\partial v} \\
S^{13}=S^{31}=\frac{v_{\phi}}{v} \frac{\partial^{2} g}{\partial v^{2}}+\left(1+\frac{v_{\phi}^{2}}{v^{2}}\right) \frac{\partial^{2} g}{\partial v \partial v_{\phi}}+\frac{v_{\phi}}{v} \frac{\partial^{2} g}{\partial v_{\phi}^{2}} \\
S^{33}=\frac{v_{\phi}^{2}}{v^{2}} \frac{\partial^{2} g}{\partial v^{2}}+\frac{\partial^{2} g}{\partial v_{\phi}^{2}}+\frac{\left(v^{2}-v_{\phi}^{2}\right)}{v^{3}} \frac{\partial g}{\partial v}+2 \frac{v_{\phi}}{v} \frac{\partial^{2} g}{\partial v \partial v_{\phi}}
\end{gathered}
$$

It is convenient to treat the problem in energy - angular momentum - space so that the diffusion coefficients just derived have to be transformed to the new velocity variables $E=\frac{1}{2} v^{2}+\Phi(\rho, z)$ and $J_{z}=\rho v_{\phi}$. Assuming an isotropic background distribution function in a corotating frame (i.e. $\vec{v}=\vec{u}+\rho \Omega \vec{e}_{\phi}$ ), we get expressions for the new diffusion coefficients, e.g.

$$
<\left(\Delta J_{z}\right)^{2}>=\left(\frac{J_{z}^{2}}{u^{2}}+\frac{\rho^{4} \Omega^{2}}{u^{2}}-\frac{2 J_{z} \rho^{2} \Omega}{u^{2}}\right) \frac{\partial^{2} g}{\partial u^{2}}
$$

Recasting the Fokker-Planck equation in flux conservation form, the flux coefficients finally emerge, e.g.

$$
\begin{aligned}
D_{J_{z} J_{z}}= & \frac{1}{2}\left(\frac{J_{z}^{2}}{u^{5}}-\frac{2 J_{z} \rho^{2} \Omega}{u^{5}}+\frac{\rho^{4} \Omega^{2}}{u^{5}}-\frac{\rho^{2}}{3 u^{3}}\right) F_{4}(u) \\
& +\frac{1}{2}\left(\frac{-J_{z}^{2}}{u^{3}}-\frac{\rho^{4} \Omega^{2}}{u^{3}}+\frac{2 J_{z} \rho^{2} \Omega}{u^{3}}+\frac{\rho^{2}}{u}\right) F_{2}(u)+\frac{1}{3} \rho^{2} E_{1}(u) .
\end{aligned}
$$

The functions $E_{i}$ and $F_{i}$ are constituent parts of the Rosenbluth potentials and their derivatives ( $c f$. Spitzer 1987). The next step is to orbit average these flux coefficients and again to transform them to more appropiate (logarythmically scaled or normalized) coordinates.

\section{The Code}

Between successive diffusion steps, a recomputation of the potential is necessary where the conservation of $f\left(q, J_{z}\right)$ ( $q$ is the adiabatic invariant) is to be considered. The final code then comprises a higher numerical resolution than was that in previous work, energy generation due to hardening of binaries, stellar evolutionary effects and a stellar mass spectrum.

\section{References}

Akiyama, K, Sugimoto, D. 1989, PASJ, 41, 991

Goodman, J. 1983, Ph.D. Thesis, Princeton University

Lupton, R.H., Gunn, J.E. 1987, AJ, 93, 1106

Rosenbluth, M.N., MacDonald, W.M., Judd, D.L. 1957, Phys. Rev., 107, 1

Spitzer, L. jr. 1987, Dynamical Evolution of Globular Clusters, Princeton Univ. Press 